\title{
Calabi-Yau Manifolds at the Interface of Math and Physics
}

\author{
by Shiu-Yuen Cheng ${ }^{*}$, Lizhen $\mathrm{Ji}^{\dagger}$, Liping Wang ${ }^{\ddagger}$, \\ and Hao $\mathrm{Xu}^{\S}$
}

There were once a young man and an older man. Their mathematics paths crossed and their names became merged into the long-lasting notion of CalabiYau manifolds.

How did this happen? Why did Calabi make his famous conjecture? How did Yau prove it? Why are Calabi-Yau manifolds so important? And how are they used? All these and other questions take time to answer and real experts to explain. They can probably be best understood through the interactions between math and physics, and that's where their true value lies. Although these questions have a long history, the future will undoubtedly bring more questions and surprises regarding Calabi-Yau manifolds.

Indeed, one must go back to more than 150 years ago, when Riemann introduced the concepts of Riemann surfaces and Riemannian metrics. His mapping theorem motivated people to endow each Riemann surface with a canonical Riemannian metric, and from there one of the greatest theorems in the history of mathematics, the uniformization theorem for Riemann surfaces and surfaces with Riemannian metrics, took shape.

What happened to higher dimensional Riemannian manifolds? What kind of geometric structures

\footnotetext{
* Mathematical Sciences Center, Tsinghua University, Beijing, China

Email: sycheng@mail.tsinghua.edu.cn

† Department of Mathematics, University of Michigan, Ann Arbor, MI, U.S.A.

Email: lji@umich.edu

‡ Higher Education Press, Beijing, China

Email: wanglp@hep.com.cn

$\S$ Department of Mathematics, University of Pittsburg, PA, U.S.A.

Email: haoxu@pitt.edu
}

could be built with them? Maybe there was not enough structure. How about adding complex structures? Would they admit canonical metrics? Or perhaps there was still not enough structure. How about Kähler manifolds-structures between complex geometry and algebraic geometry? This was what Calabi asked 60 years ago, and what Yau answered 24 years later. He cracked a geometric problem by unraveling hard nonlinear differential equations.

The Calabi-Yau metric has been an invaluable tool in differential and algebraic geometry. Here is an incomplete list of long-standing conjectures and problems solved by using the Calabi-Yau metric and Yau's technique of the a priori estimate.

1. The Severi conjecture, that every complex surface that is homotopic to the complex projective plane $\mathbb{C} P^{2}$ is biholomorphic to $\mathbb{C} P^{2}$.

2. Bogomolov-Miyaoka-Yau Chern number inequalities and their associated uniformization theorems in higher dimensions.

3. Uniqueness of Kähler complex structure on $\mathbb{C} P^{n}$.

4. Torelli theorem for K3 surfaces.

5. Every K3 surface is Kähler.

6. The existence of Hermitian-Yang-Mills connections in stable vector bundles (DonaldsonUhlenbeck-Yau theorem).

7. The existence of complete Kähler-Einstein metrics on pseudoconvex domains.

8. Calabi-Yau manifolds are used in the context of string theory to make models of particle physics unified with quantum gravity.

9. Construction of non-flat compact simplyconnected Kähler manifolds whose Ricci curvature is identically zero. 
10. Any compact Kähler manifold with non-negative first Chern class is covered holomorphically by the product of $\mathbb{C}^{n}$ and a compact simply connected Kähler manifold.

11. The vanishing of $c_{1}$ and $c_{2} \cup[\omega]^{n-2}$ for a Kähler manifold implies that the manifold admits a metric with zero curvature and is covered by the flat torus.

12. As conjectured by Yau, the existence of KählerEinstein metrics on Fano manifolds has intimate connections to the stability of manifolds. Important progress has been made recently.

Such beautiful results are appreciated not only by mathematicians but by physicists as well. For, according to string theory, Calabi-Yau manifolds provide the hidden inner spaces of our universe.

It is true that 60 is a magic number, but 65 is also a milestone in one's life. Here is a poem by Professor Yau reflecting his experience with geometry and physics.

Forty years of geometry, equation creates structure, analysis blends perfection and beauty.

Thirty years of physics, strings and branes drifting through

hyperspace,

breaking supersymmetry as they go.

Quantum connects to gravity, and nature is whole again.

S.-T. Yau

\section{The Power of Calabi-Yau Manifolds}

From the Uniformization (Klein, Poincare, Koebe, Brouwer) to Riemman-Hilbert Correspondence, Picard-Fuchs Equations, and Kähler-Einstein Metrics (Calabi-Yau)

1. Number theory: Calabi-Yau manifolds are natural, higher-dimensional generalizations of elliptic curves - the theory of which dominated number theory in the 20th century. Many believe that Calabi-Yau manifolds will be the main tool in number theory in the 21st century. The related questions of modular forms and quasi-modular forms are now being developed.

2. Algebra: There is a natural category, which appeared in algebra, where the Calabi-Yau condition plays an important role. It is used in classifying topological field theories.

3. Differential geometry: The construction of the Calabi-Yau metric solves the old problem of classification of manifolds with holonomy group $S U(n)$ and
$S P(n)$ by reducing the construction of them to algebraic conditions.

$$
\text { 4. Algebraic geometry: }
$$

(a) The second Chern class of such manifolds is numerically positive in the sense that the cup product of it with the top number of products of any positive $(1,1)$ class is positive unless it is covered by the torus.

(b) Calabi-Yau manifolds are stable and their tangent bundles are also stable with respect to any polarization.

(c) Counting the number of algebraic curves in Calabi-Yau manifolds has become quite successful since the introduction of the concept of mirror symmetry, which has opened up many new directions in mathematics.

(d) It gives a structure theorem for Compact Kähler manifolds with non-negative first Chern class.

5. Nonlinear partial differential equations: The proof of Calabi conjecture gave the first general method to solve the nonlinear Complex Ampere equation on a complex manifold. The equation solves a very important inverse problem: given any volume element on any compact Kähler manifold, any Kähler metric can be deformed to a new Kähler metric whose volume form is the given one up to a constant. The methods of estimates have been fundamental to all constructions of Kähler-Einstein metrics, including Kähler metrics with prescribed singularities. The singular metrics were used by Donaldson et al. to solve a famous conjecture of Yau on existence of KählerEinstein metric on Stable Fano manifolds.

\section{Perspectives of Experts}

When Yau turned 65 this year, we asked experts all over the world, in both math and physics, to comment on the applications and impacts of Calabi-Yau manifolds and on the work of Yau. What followed is the mathematical garden of Yau and Calabi-Yau manifolds as seen through their eyes. We hope you will enjoy a walk through this joyful, colorful, and mindexpanding garden.

\section{In Mathematics...}

Calabi-Yau manifold is the treasure of human civilization, although many of its mysteries are yet to be explored. In view of its surrounding theorems, tools, questions and conjectures, it has been an extremely active and important research field.

Lo Yang

Academy of Mathematics and System Sciences, Chinese Academy of Sciences (Hua Loo-Keng Mathematics Prize 1997) 
There is no doubt that Calabi-Yau manifolds have become justly famous. The mere fact that they are now abbreviated to CY manifolds testifies to their importance.

In both geometry and physics the Kummer surface and its relatives have been of central interest since the 19th century. They are the natural successors to elliptic curves, and look how long these curves have fascinated mathematicians. Yau has pioneered many important ideas in global geometric analysis and has influenced much of the mathematics and physics of our time.

Michael Atiyah University of Edinburgh (Fields Medal 1966; Abel Prize 2004; King Faisal International Prize 1987)

Calabi-Yau manifolds form a very specific class of mathematical objects, which play an increasingly important role in various branches of math and physics. They are fundamental in algebraic geometry, where they stand somehow in the middle of the complexity hierarchy of algebraic varieties: not too simple, but still accessible. In differential geometry they provide the simplest examples of the so-called Kähler-Einstein manifolds, a very particular and very rich structure. They are fundamental in math physics, in particular in superstring theory: they are the basic objects of Mirror Symmetry, a surprising duality phenomenon which is one of the major discoveries of the last 30 years in theoretical physics.

Arnaud Beauville University of Nice, France

I first met Shing-Tung Yau when he joined the Stanford Mathematics Department in 1972. It was immediately clear that he had a remarkable talent and passion for mathematics, not just in his own field of differential geometry, but across the whole spectrum of deep open problems in mathematics. When hearing him talk about mathematics or Chinese civilisation in those days, his many ideas are perhaps best described by the line from Po Chu-i: "Like pearls large and small cascading on a plate of jade."

Yau's whole career since Stanford has borne out the immense promise which was then apparent to all of us who knew him well. His solution of the Calabi conjecture has brought into mathematics a class of complex manifolds which seem likely over the next century to take over the central role played by elliptic curves in the evolution of mathematics in the 19th and 20th century. Indeed, Calabi-Yau manifolds have already been shown to be of great importance in physics, and one can speculate that their arithmetic mysteries, still largely untouched by number-theorists, could some day throw light on even some of the most intractable ancient problems of the subject.

In addition to his own mathematical research, Yau has played an immense role since the middle 1990's in the rebuilding of mathematics in China. His creation and organization of the ICCM's has brought together mathematicians of Chinese origin from all over the world, and built up a new esprit de corps amongst them. He has known how to persuade the highest political voices in China that high-level mathematical research is of great importance for the future of China, and deserves good financial support. Above all, Yau has striven always to foster the very finest mathematical research, and to give gifted young Chinese mathematicians every chance to develop their talents. Just one of many examples of Yau's legacy in this direction, which I enormously appreciate, has been his creation of an outstanding group of numbertheorists in the Chinese Academy of Sciences in Beijing. It seems no exaggeration to say that Yau's myriad contributions to all aspects of the mathematical world must place him amongst the greatest scholars in the long and rich intellectual history of China.

John Coates

Cambridge University (Senior Whitehead Prize 1997)

The notion of Calabi-Yau manifolds has been generalized to algebras (and categories), and they are used in classifying topological field theories.

\section{Ralph Cohen Stanford University}

Calabi's conjecture from the 1950's, concerning metrics with prescribed Ricci tensor on compact complex manifolds, and Yau's verification of the conjecture from the 1970's are historic landmarks in differential geometry. The verified conjecture yields the first examples of non-flat compact manifolds with vanishing Ricci curvature; answering a fundamental question in global Riemannian geometry. More generally, the nature of the conjecture and its solution-involving a deep interaction between nonlinear elliptic partial differential equations and differential geometry-signalled a radical new development which has pervaded the field in the ensuing 40 years. At a detailed, technical, level, the crucial ideas and estimates for the complex Monge-Ampère equation introduced by Yau at 
that time are still being refined and exploited in current developments.

The study of elliptic curves goes back to the 18th century origins of algebraic geometry and complex analysis. By the middle of the 20th century, many of the special features of K3 surfaces had emerged. Nowadays, with inspiration from string theory in theoretical physics and from developments in algebraic geometry, we see at least a glimpse of the extraordinary and beautiful properties of Calabi-Yau manifolds in all dimensions. A huge amount is known but there are profound questions and mysteries which will surely occupy generations of geometers to come.

Simon Donaldson Stony Brook University (Fields Medal 1990; Crafoord Prize 1994; Shaw Prize 2009; Breakthrough Prize in Mathematics

For me, the main "application" of Calabi-Yau manifolds is that they are very mysterious and challenging. In fact, this is true already for K3 surfaces. (As far as I know, one of the reasons for the name K3 was to hint that these surfaces are even less accessible than the famous mountain $\mathrm{K} 2$ in the Himalayas.) E.g., the proof of the Torelli theorem for K3 surfaces is mysterious (I doubt that we know a direct way of constructing a K3 surface from its periods). The existence of the Calabi-Yau metric on a K3 surface (e.g., on a quartic in P3) is again a mystery for me: already in this particular case one needed the power of Geometric Analysis (and the intellectual power of Prof. Yau) to construct it.

\section{Vladimir Drinfeld Yale University}

(Fields Medal 1990)

Elliptic curves have a long history. In complex geometry, in algebraic complex geometry, their moduli, the $\mathrm{j}$-line is a fundamental invariant. In arithmetic geometry, the Birch and SwinnertonDyer conjectures predicts a property of the Lfunction of elliptic curves over a number field. In positive characteristic, over a finite field, Hasse proved for elliptic curves what later was called the Weil conjectures. K3 surfaces have also a long history, even if shorter. In complex geometry, in algebraic complex geometry, their moduli, notably the Torelli theorem (Siu), are fundamental objects and theorems. I do not know about properties of the L-function over number fields. But over finite fields, Deligne proved first the Weil conjectures for K3 surfaces before he could prove them in general.
Ogus studied the Torelli theorem in positive characteristic, and recently (Madapusi Pera and others) proved the Tate conjecture for divisors in positive characteristic. Those are just a few examples of the role of elliptic curves and K3 surfaces in algebraic and arithmetic geometry. As we know, Calabi-Yau varieties generalize to higher dimension elliptic curves and K3 surfaces. We do not know much about their moduli or Torelli theorem, nor do we know much about their $L$-functions. In positive characteristic, their lift to characteristic 0 is not even known, it is also a question of what definition one takes in positive characteristic. So the field is quite open.

\section{Hélène Esnault \\ Freie Universität Berlin \\ (Gottfried Wilhelm Leibniz Prize 2003)}

I think that the Calabi-Yau theorem and the Donaldson-Uhlenbeck-Yau theorem are the two most important theorems in complex geometry in 20 th century after Kodaira's work. Using these two theorems, one can study the moduli spaces of CY manifolds and of stable vector bundles. On the other hand, nowadays these two theorems are still very useful. For example, Demailly and Paun used the CY theorem to study the Kähler cone and got a very deep theorem. Voison used the DonaldsonUhlenbeck-Yau theorem to prove that the Hodge conjecture is false on the Kähler manifold.

As to the applications of estimates of Yau's paper on Calabi conjecture, I mention only one paper. In 2008, Boucksom, Eyssidieux, Guegj, and Zeriahi wrote a very important paper, "Monge-Ampère equations in big cohomolody classes." In Sections 5 and 6 of that paper, the authors made numerous citations of Yau's estimates.

Jixiang Fu

Fudan University

(Morningside Silver Medal 2010)

The impact of Calabi-Yau manifolds on mathematics-number theory, algebraic and differential geometry, symplectic geometry, and theoretical physics-has been enormous. For example, in number theory, Calabi-Yau manifolds are natural generalizations of abelian varieties and K3 surfaces, and there is a lot of work trying to match their zeta functions over number fields with Lfunctions coming from automorphic forms.

Benedict Gross

Harvard University (Cole Prize 1987) 
Certainly Calabi-Yau manifolds have been widely important. In algebraic geometry, they are one of the key classes of varieties in classification theory, and many mysteries remain. They are crucial in string theory, providing the compactification of space-time to ten dimensions. As a result, there has been a great deal of influence of string theory on algebraic geometry, especially through mirror symmetry. The Strominger-Yau-Zaslow conjecture also introduced differential geometric ideas into mirror symmetry, and has a strong influence on a great deal of work, even though it remains unresolved.

\section{Mark Gross Cambridge University}

It is hard to overstate the impact that CalabiYau manifolds have had in both mathematics and physics. Yau's positive solution of the Calabi conjecture shows the existence of Ricci-flat Kähler metrics on Kähler manifolds with vanishing real first Chern class. This result is what fundamentally enabled the use of Calabi-Yau manifolds for string compactification. Progress in the geometry of Calabi-Yau manifolds and their moduli has been paralleled by similar progress in string theory, leading to the rich cross-fertilization between mathematics and physics that is still going strong 30 years later.

Yau has been a major player in the development of this field over the decades, in mathematics, in physics, and in the hybrid offsping of these two disciplines. His contributions are vast and seminal, extending over a broad swath of the field including work on Gromov-Witten invariants, PicardFuchs systems, mirror symmetry, the A-model, elliptic fibred Calabi-Yau manifolds, heterotic string theory, F-theory, and much more. I also mention in this context his celebrated construction with Uhlenbeck of Hermitian-Yang-Mills connections on stable bundles, which has also played a fundamental role in the geometry/physics collaboration, extending beyond Calabi-Yau manifolds and string theory.

Yau's work has both informed and inspired my own work and that of many others, and continues to do so. I am happy to join my colleagues in this tribute to Yau on the occasion of his 65th birthday.

\section{Sheldon Katz University of Illinois at Urbana-Champaign}

The most striking applications of Calabi-Yau manifolds are in string theory. In mathematics the CY theorem has inspired the study of special metrics in many other contexts: holomorphic vector bundles, Fano manifolds, special Lagrangian submanifolds, G2 and Spin(7) manifolds. Special metrics and algebro-geometric stability enjoy a close and mutually beneficial relationship. Special metrics lead to Hausdorff moduli spaces. Another important feature of CY manifolds is the smoothness of the corresponding moduli spaces.

David Kazhdan

Hebrew University of Jerusalem and Harvard University

(Israel Prize 2002)

Calabi-Yau manifolds are Einstein manifolds (i.e. zero Ricci curvature) with compatible complex structures. Yau's theorem gives a complete characterization of such manifolds in the compact setting. In string theory, they are internal spaces whose geometry determines the physics of our four-dimensional spacetime. Mirror symmetry for Calabi-Yau manifolds is a duality between two seemingly very different types of geometry, namely complex geometry and symplectic geometry. The Strominger-Yau-Zaslow conjecture unveils the mystery of mirror symmetry as a geometric Fourier transformation along special Lagrangian fibrations. It has far reaching implications in almost all branches of geometry, topology and beyond. They include enumerative geometry, gauge theory, calibration theory, toric geometry, affine geometry, hyperkähler geometry, geometry of special holonomy, knot theory and even geometric Langlands in number theory.

\section{Conan Leung Chinese University of Hong Kong (Chern Prize 2010)}

The beauty of the geometry of Calabi-Yau manifolds lies in its mirror symmetry of the algebraic geometry of the algebraic structures of these manifolds on one hand, and of the differential geometry arising from the Calabi-Yau metrics of these manifolds on the other hand. The study of Mirror Symmetry of Calabi-Yau manifolds, like a "gold rush," has provided both mathematicians and mathematical physicists a golden opportunity to reveal the inner beauty of the space we imagined ourselves to have lived in.

Jun Li

Stanford University (Morningside Gold Medal 2001)

Professor Yau has a deep and broad understanding about mathematics as a whole, and he pays 
a lot of attention to the development of applied mathematics as well. This is clear from the speakers he has invited for important events like ICCM and the inaugural celebration for the Sanya Math Forum. Personally I am grateful to his strong support of number theory, which is not his major field of research. For example, in each ICCM, there are number theorists receiving the Morningside medals (gold or silver). Behind the scene, Prof. Yau is a strong supporter of Yitang Zhang's various awards, including his induction to the Academy of Academia Sinica in Taiwan. Different from many other well-established top mathematicians in the world, Professor Yau pays a lot of attention to math education. He initiated various levels of mathematics competitions, from high school to Ph.D. students, open to students of Chinese origin all over the world. He is the greatest fund raiser among all mathematicians. He has founded several math and applied math centers, raised funds to build new buildings to house many math institutes and centers and to finance their operations, to support the ICCM, and to offer competition awards, etc. He has educated many outstanding mathematicians. His influence and impact on the advancement of mathematics and on the Chinese math community are far-reaching. Some of my Indian friends are very envious of the Chinese, as they feel sorry about the lack of an Indian math leader like Yau.

Winnie Li

Pennsylvania State University (Chern Prize 2010)

The Estimate of Yau on the complex MongeAmpère equations is the only global estimate available even now, after its establishment more than three decades ago. Its impact on algebraic geometry is profound, particularly in the classification of minimal models with singularities.

\section{Chang-Shou Lin Taiwan University (Morningside Gold Medal 1998)}

Calabi-Yau has influenced almost all major areas of mathematics, from number theory, algebra, algebraic geometry, topology, geometric analysis and partial differential equations. Calabi-Yau has become a household name in mathematics and in the real world. Almost every day we hear lectures about Calabi-Yau manifolds, Calabi-Yau algebras, Calabi-Yau categories, Calabo-Yau metrics and Calabi-Yau equations, to mention a few. Calabi-Yau manifolds and their moduli spaces are among the most beautiful and most mysterious mathematical objects I have ever seen.

$$
\begin{array}{r}
\text { Kefeng Liu } \\
\text { University of California, Los Angeles } \\
\text { and Zhejiang University } \\
\text { (Morningside Gold Medal 2004) }
\end{array}
$$

Calabi-Yau manifolds in the strict sense are complex Kähler manifolds, the special unitary group as holonomy. The Calabi conjecture (as proved by Yau) tells us (among other things) that there are plenty of them; for a compact Kähler manifold with trivial canonical bundle we can modify the Kähler metric within its cohomology class such that it becomes a Calabi-Yau manifold. This theorem has (and continues to have) far reaching consequences outside differential geometry (to which it belongs): it permeates algebraic geometry as well as mathematical physics. For instance, this led to a simple and short proof that the Torelli map for Kähler K3 surface is surjective. But its impact is being even more strongly felt in the mathematics surrounding Mirror Symmetry, for it is there the central notion. Indeed, via Mirror Symmetry this has become so ubiquitous that virtually every person considering him or herself an intellectual has heard it pronounced or seen it written.

\section{Eduard Looijenga Tsinghua University}

Calabi-Yau manifolds belong to the intersection of several geometric worlds (complex, symplectic, and Riemannian geometry) and a world of theoretical physics (quantum string theory). They have an incredible amount of beautiful properties, even if one restricts oneself to the dimension three.

On various scales, our understanding of the physical world is dominated by several basic pictures. We live in Euclidean 3-dim space on a human scale. We live in a (pseudo-)Riemannian Einsteinian 4-dim space-time on a cosmological scale. And on the scales of Planck size and lower, our world is filled with minuscule Calabi-Yau threefolds, determining physics of the observable matter.

Yuri Manin
Max-Planck-Institut für Mathematik, Bonn,
Germany and Northwestern University
(Nemmers Prize in Mathematics 1994;
Schock Prize 1999; The King Faisal International
Prize of Science 2002)

S.-T Yau's proof of the Calabi conjecture stands as the foundation for much subsequent work, in understanding the structure of Kähler manifolds. 
These manifolds lie at the crossroads of Riemannian, complex analytic and algebraic geometry. Of singular importance is the subset of Kähler manifolds now known as Calabi-Yau manifolds. These also play a central role in arithmetic geometry, symplectic geometry and string theoretic physics. For example, three-dimensional CY-manifolds provide-amongst many other things - the original geometries contributing to the rich field of "mirror symmetry," a field that has profoundly influenced enumerative algebraic geometry, symplectic geometry, and string theory.

Barry Mazur (Veblen Prize 1966; Cole Prize 1982; National Medal of Science 2011)

Peter Kronheimer (Veblen Prize 2007) Clifford Taubes (Veblen Prize 1991;

Shaw Prize 2009)

Harvard University

In algebraic geometry and complex analytic geometry, the theory of Calabi-Yau manifolds is a special and important branch. First of all, CalabiYau manifolds constitute one of the five fundamental elements (the remaining four are rationally connected manifolds, complex tori, complex symplectic manifold and manifolds of general type). Second, the manifolds appear as the invisible 6 dimension of our universe in the theory of super strings, which is anticipated to be able to systematically explain the known four forces in physics. Finally, the intrinsic structure of Calabi-Yau manifolds is so mysterious. So far, there are so many examples that we cannot be sure if there are only finitely many deformation types; although counting rational curves on a given Calabi-Yau manifold is an essential recipe in super string theory, we do not know in general if it carries a single rational curve.

Yoichi Miyaoka University of Tokyo

The existence of Calabi-Yau manifolds, established by Professor Yau in his famous solution of the Calabi conjecture, is one of the most important landmarks in modern mathematics. It is a landmark in the theory of partial differential equations, as it provided the first solution of a full-fledged complex Monge-Ampère equation. It is a landmark in differential geometry, as it is the first significant generalization of the Uniformization Theorem of nineteenth-century mathematics. It is a landmark in algebraic geometry, as it opened the way to the moduli theory of the most important and natural generalizations of elliptic curves. And it is a landmark in mathematical physics, as Calabi-Yau manifolds also arise as vacuum configurations of superstrings. It is hard to think of any other breakthrough which has had so much impact and on so many fields of mathematics.

Duong Phong Columbia University (Bergman Prize 2009)

The study of Calabi-Yau manifolds has engaged a vast array of mathematicians and physicists, leading to fantastic new insights in algebraic, differential, and symplectic geometry, category theory, and mathematical physics, over the past three decades. The beautiful properties of their Ricci-flat metrics lead to their primordial role in string theory, motivating the new domain of mirror symmetry. This is now one of the most productive areas of investigation in mathematics, encompassing the theory of pseudo-holomorphic curves and Gromov-Witten invariants, Fukaya categories in symplectic geometry, moduli spaces of Bridgeland stability conditions, character varieties, the study of derived categories, modular forms, and many other things. Major conjectures such as Kontsevich's Homological Mirror Symmetry approach are strongly motivating a wide mathematical community.

Carlos Simpson University of Nice, France

Before Yau's proof of the Calabi conjecture, almost nothing was known about Calabi-Yau manifolds, and mathematicians had no tools for studying them. His proof has made possible things never dreamt of beforehand. Any result we now know about these manifolds uses Yau's theorem in its proof.

Most importantly, the result created new links between different areas of mathematics, leading to whole new subjects that did not exist before 1980 . Many of these new ideas come from "mirror symmetry"-a series of breathtaking predictions from string theory that have dominated large parts of modern geometry in the last 25 years. Yau's theorem provides the links to string theory by solving Einstein's equation on Calabi-Yau manifolds, and has also provided our best approach to solving mirror symmetry, using special Lagrangian submanifolds and the Strominger-Yau-Zaslow conjecture.

Richard Thomas

Imperial College of London 
Prof. Yau's proof of the Calabi Conjecture is a true gem of mathematics. It introduced the use of Moser iteration to obtain uniform estimates for solutions of geometric PDEs; he then obtained a second-order estimate for the solution which surprisingly did not depend on a gradient bound, but only on the uniform estimate; and then in a true tour de force, he derived third-order derivative bounds for the solution, using the second-order bounds. All of these techniques were groundbreaking, and have since then become part of the standard toolbox of any complex geometer and used countless times, to the point that some people nowadays forget that they originated in Prof. Yau's work.

\section{Valentino Tosatti Northwestern University}

What I find amazing about Calabi-Yau manifolds is their impact in physics and the resultant modelling of the universe by ten-dimensional space. At first this seems bizarre but I imagine that early in the last century relativity theory and fourdimensional space-time was also a great conceptual leap for science, so it would be very interesting to look back on Calabi-Yau manifolds in the decades ahead.

Neil Trudinger

Australia National University

(Australian Mathematical Society Medal 1981)

Calabi-Yau manifolds are central in algebraic geometry, because they appear naturally as fibers of the Iitaka fibration of any variety and they sit between two extreme classes, namely varieties of general type, with many holomorphic contravariant tensors, and rationally connected varieties, containing big families of rational curves. Calabi-Yau geometry is beautiful and still mysteriously related to mathematical physics (mirror symmetry) and also to symplectic geometry. Indeed, varieties of general type tend to have trivial Gromov-Witten invariants, while in the rationally connected case, the quantum cohomology corrections tend to be polynomial; in the CY case, the Gromov-Witten potential is actually an infinite series, showing that the study of rational curves in CY manifolds is very rich. The work of Yau establishing the existence of Kähler-Einstein metrics and the stability of their tangent bundle was the starting point for many further progresses, but a number of big questions remain open, both on the analytic geometry and on the algebraic geometry sides. One of them is the relation between the Kodaira dimension zero and the Calabi-Yau property. Another one concerns the classification of $\mathrm{CY}$ varieties: Are there infinitely many families in a given dimension? This is unknown in dimension 3, and also for hyper-Kähler manifolds.

Claire Voisin University of Paris VI and École Polytechnique (EMS Prize 1992; Sophie Germain Prize 2003; Satter Prize 2007; Clay Research Award 2008)

Being the potential candidate as the inner space in string theory, as well as the building block towards the classification of higher dimensional algebraic varieties, Calabi-Yau manifolds appear to be the most fundamental and influential geometric objects after Yau's resolution of the Calabi conjecture in 1976. Even with many insightful discoveries done by many mathematicians and physicists in the past thirty or more years, it is believed that a significant part of 21 st century mathematics will be devoted to uncovering the underlying mysteries of Calabi-Yau spaces.

Chin-Lung Wang Taiwan University

(Morningside Silver Medal 2001)

Ever since the rise of mirror symmetry some twenty years ago, Calabi-Yau manifolds have been established as one of the most versatile subjects in mathematics and physics.

Spectacular results have been obtained in many different areas such as arithmetic, algebraic, differential and symplectic geometry, differential (Picard-Fuchs) equations, and physics (string theory), with unmistakable focus of research on Calabi-Yau manifolds.

There are numerous examples in which CalabiYau manifolds are encountered at the junction of two or more different fields. For instance, the list of relations with physics starts with the unexpected relevance of algebraic varieties as compactification manifolds for string theory, continues with the even less expected enumerative predicts resulting from mirror symmetry, and can be extended all the way to the present day and to the future.

Noriko Yui University of Toronto

To appreciate the impact of Yau's solution of the Calabi conjecture, we should remember that before the work of Yau, nonlinear PDE was a difficult subject in analysis hardly related to algebraic geometry. Such a link was established only through the 
insight and technical power of Yau. It gave great inspiration to people working in the field. As an example, Donaldson, in his essay in the book Fields Medalists' Lectures, wrote (after Hitchin suggested to him the thesis problem of Hitchin-Kobayashi correspondence): "I soon began to tinker with analytical approaches to Hitchin's conjecture, perhaps motivated by the renowned work of Yau, a few years before, on the Calabi conjecture." Since then, nonlinear analysis has been applied to solve many important problems in disciplines other than algebraic geometry. On the other hand, Calabi conjecture (Yau's theorem) continues to play important roles in recent developments in complex geometry. Here, we should also point out that even in situations where Yau's theorem does not apply directly, the estimates established in his fundamental paper "On the Ricci curvature of a compact Kähler manifold and the complex Monge-Ampère equation" have found numerous applications. A recent example is the work of Chen-Donaldon-Sun verifying a conjecture of Yau relating stability to the existence of KE metrics on a Fano manifold.

Xiping Zhu

Sun Yat-sen University

(Morningside Silver Medal 2004)

\section{In Physics...}

Shing-Tung Yau is one of the most influential people in the international mathematics community. His long-lasting influence on the young generation of Chinese mathematicians and on the Chinese mathematics community is of historical scope.

Chen-Ning Yang

Tsinghua University

(Nobel Prize in Physics 1957; National Medal of Science 1986; Benjamin Franklin Medal 1993; Einstein Medal 1995)

It is hard to overstate the importance Calabi-Yau manifolds in physics. String compactifications on Calabi-Yau's are the canonical paradigm for connecting string theory to reality, showing that seemingly random and complicated four dimensional particles and interactions of the sort we see in the real world can arise in a simple and striking way from a beautiful higher-dimensional geometry. The study of string theory on Calabi-Yau's has also yielded deep clues into the inner workings of the theory itself, ranging from conifolds to mirror symmetry to twistor string theory. One can only expect this trend to continue in the coming years.

Nima Arkani-Hamed Institute for Advanced Study, Princeton

(Gribov Medal of the European Physical Society 2003; Fundamental Physics Prize 2012)

The two pillars on which our understanding of nature is based are Einstein's theory of general relativity, which is used to describe gravity, and quantum field theory, which describes the non-gravitational forces. These theories contradict each other, and a fundamental problem in modern theoretical physics is the incorporation of Einstein's theory into quantum field theory. Such a theory is known as quantum gravity, and the leading theory of quantum gravity is string theory. Calabi-Yau manifolds figure prominently in string theory. It has been possible to show not only that string theory unifies gravity and quantum field theory but that particles like electrons and quarks and known forces can arise. And so it is a candidate as a unified theory of all forces. The needed constructions are based on Calabi-Yau manifolds.

\section{Melanie Becker and Katrin Becker Texas A\&M University}

I would say that CY manifolds play an essential role in connecting string theory with the observed 4 dimensional world and with elementary particle physics. Without CY manifolds there would be very little interest in string theory and the many advances in mathematics that are due to connections between mathematics and string theory would not have been made.

Philip Candelas University of Oxford

Over the past 20 years our understanding of Calabi-Yau manifolds has grown from rudimentary to rich. Shing-Tung Yau's contributions have shaped the study of these important manifolds from the start. His solution to Calabi's conjecture established the existence of a unique Ricciflat Kaehler-Einstein metric in each Kähler class on compact manifolds with trivial canonical bundle. Yau's approach was famously non-constructive; to this day there is no known closed-form expression for this metric on any of the hundreds of millions of (families of) known examples. Fortunately for both physics and mathematics, when stringtheorists discovered the importance of Calabi-Yau manifolds in the mid-1980's as part of their quest to create a theory of quantum gravity, the existence of the Calabi-Yau metric proved sufficient for 
this critical application. It is instead the topological and Hodge-theoretic features of Calabi-Yau manifolds which are crucial for physical application. The interplay between the Hodge-theoretic properties and moduli of Calabi-Yau manifolds, often with string-theoretic motivation (e.g., "B-model" geometry in mirror symmetry), has been a consistent theme of research at the math/strings interface ever since. From his early studies of the mirror map through his recent work on tautological local systems, Shing-Tung Yau has continually led the way. In large part through his efforts, CalabiYau manifolds have gone from being little understood curiosities to geometric gems of the first order within a single generation.

Charles Doran University of Alberta

Superstring theory, the leading candidate for a theory of fundamental interactions, is formulated in ten dimensions. If superstring theory describes our universe, six of these dimensions must be in the form of a very small compact manifold, whose metric must solve Einstein's equation. Calabi-Yau manifolds were the first proposal for such solutions, and though others have been found since, they are still the best motivated and leading proposal. Their topology and geometry leads directly to the spectrum and properties of particle physics in four dimensions, so someday this proposal may find experimental verification.

Michael R. Douglas Simons Center, Stony brook (Sackler Prize 2000)

Prof. Yau has suffused the mandate of mathematics, and specifically of algebraic geometry, into the rules of physics to an unprecedented degree. By his contributions to human understanding of our place in nature and reality, he has earned the greatest respect and honor in both fields.

\section{James Gates University of Maryland (National Medal of Science 2013; Mendel Medal 2013)}

The solution by Prof Yau of the Calabi conjecture has led to important advances in both Algebraic and Differential Geometry and to a revolution in the picture of the world provided by Super String Theory. Calabi-Yau spaces provide the richest store of Ricci flat manifolds, and manifolds of special holonomy and these special properties are essential for their current role in phenomenologically viable compactification scenarios and in applications to supersymmetric quantum field theories. It is difficult to think what modern theoretical physics would be without them.

\section{Gary Gibbons \\ Cambridge University}

One of the most amazing developments in theoretical physics in recent decades is the realization that the beautiful geometrical manifolds constructed by E. Calabi and S. T. Yau provided the framework for solutions of 10 dimensional superstring theory wherein 6 of the spatial dimensions are compactified to form a Calabi-Yau manifold. In a manner that would have pleased the Greek philosophers and mathematicians, the nature of matter and the structure of forces is dictated, in Calabi-Yau compactifications, by the geometrical properties of these manifolds. The study of these solutions and these manifolds has deeply influenced developments in both physics and mathematics for the last thirty years.

\section{David Gross \\ University of California, Santa Barbara (Dirac Medal 1988; Harvey Prize 2000; Nobel Prize in Physics 2004)}

As a physicist I am always amazed by the great impact of mathematics on physics. There have been at least five profound revolutions in the history of physics, and a sixth is now in progress:

(1) The revolution of Newtononian mechanics in the 17th century, for which Newton invented calculus as its mathematical foundation.

(2) The revolution of Einstein's special relativity theory in 1905, which unified Newtonian mechanics with the Maxwell equations of electromagnetism, and is formulated by the Lorentz group in mathematics.

(3) The revolution of quantum mechanics (from about 1900 to the 1920s), which is mathematically based upon Hilbert space and linear algebra.

(4) The revolution of Einstein's general relativity (about 1916) which is deeply rooted in Riemann geometry and opens up the era of modern cosmology.

(5) The revolution, from the 1940s to the 1970s, of quantum field theory, especially the standard model, which unifies special relativity with quantum mechanics, describing electromagnetic, weak, and strong forces in Nature. Its mathematical foundation includes functional path integrals, gauge groups, and fiber bundles.

(6) The current greatest challenge in physics is to unify gravitational force with the other three 
forces of nature, and to understand the origin of space-time itself. This definitely calls for a profound new revolution. The leading candidate for such a revolution is the ten-dimensional string theory or eleven-dimensional M-theory, containing hidden 6D Calabi-Yau manifolds, as introduced in the 1980s and 1990s. The impact of Calabi-Yau on modern string theory is far-reaching, similar to the role of Riemann geometry in general relativity. Professor Yau has been standing at the center of these exciting developments since then. He has built up the mathematical foundation, and, in cooperation with prominent physicists, is in the leading position at the interface of mathematics with physics today, at the dawn of the 21 st century.

Hong-Jian He Tsinghua University

Calabi-Yau spaces have had a huge impact in physics. They first gained prominence with the realization that compactifying string theory on one of these geometries gives rise to physics very close to the standard model. It was later shown that singularities in Calabi-Yau spaces can give rise to nonabelian gauge symmetries like the ones seen in physics. Calabi-Yau spaces were also used to give one of the first examples of topology change in quantum gravity. Physicists continue to explore new applications of these remarkable geometries.

Gary Horowitz University of California, Santa Barbara (Xanthopoulos Prize 1993)

The investigation of the algebraic topology of Calabi-Yau manifolds has been at the very heart of many of the most striking developments in physical mathematics for the past thirty years. In addition to the important role it has played in string compactification, and in efforts to make realistic models of string theory, investigations into CalabiYau compactifications led to the momentous discoveries of mirror symmetry and homological mirror symmetry. The far-reaching ramifications of these discoveries are still being worked out to this day.

Gregory Moore Rutgers University

(Dannie Heineman Prize 2014)

Einstein's vision of physical laws emerging from the shape of spacetime has been expanded by the higher dimensions of string theory. This vision has transformed not only modern physics, but also modern mathematics. By proving the existence and uniqueness of a large class of solutions to the higher dimensional Einstein equations, Shing-Tung Yau brought these two fields together. This work has had countless implications, from laying out the landscape of string vacua to the discovery of mirror symmetry, in which string theory modifies the basic concepts of geometry.

Joseph Polchinski

University of California, Santa Barbara

(Dirac Medal 2008; Physics Frontier Prize 2013)

Calabi-Yau manifolds were first used in string theory in the context of construction of semirealistic compactification of heterotic string theory. Since then they have played a central role in many aspects of string theory. Some of the examples I can mention are mirror symmetry, topological string theory, string dualities, and black holes. They continue to play a major role in string theory today and this is likely to continue in the future.

Ashoke Sen

Harish-Chandra Research Institute, Allahabad (Fundamental Physics Prize 2012;

Dirac Medal 2014)

I am sure that many mathematicians and string theorists will comment on the extremely significant role Calabi-Yau manifolds play in mathematics and string theory. Therefore, I would like to focus on a more narrow aspect-their impact on quantum field theories. A two-dimensional non-linear sigma model with $(2,2)$ supersymmetry, whose target space is a Calabi-Yau manifold is a very interesting quantum field theory. In addition to its supersymmetry, it is also conformally invariant. Such theories are extremely rich, exhibiting many important phenomena, some of which are exactly calculable.

Therefore, they are a useful laboratory teaching us about more generic properties of quantum field theories. Lessons extracted from this laboratory have already revolutionized our general understanding of quantum field theory. Examples are the role of exactly marginal deformations, the chiral ring, mirror symmetry/duality, topological field theory, etc. I have no doubt that the study of such quantum field theories will continue to be productive and that it will inspire many more insights.

Nathan Seiberg Institute for Advanced Study, Princeton (Heineman Prize 1998; Fundamental Physics Prize 2012)

Over the last thirty years Calabi-Yau manifolds have come to play a central role in the search by 
physicists for a unified theory of nature. Compactification of ten-dimensional superstring theory on a Calabi-Yau threefold yields a four dimensional universe with properties remarkably like the one we inhabit. A wealth of both mathematical and physical insights have been gained from ongoing analyses of the rich structure of these compactifications.

Andrew Strominger

Harvard University

(Physics Frontiers Prize 2013)

The counterpart of a DNA code in physics is a Calabi-Yau manifold with branes and fluxes-or other data needed to describe a compactification or a background.

Leonard Susskind Stanford University (Sakurai Prize 1998)

Calabi-Yau manifolds have been the source of many new and deep ideas in string theory. Not only have they provided us with concrete mathematical objects which are starting points of extracting particle physics data from string theory, but also, in the context of topological strings they have led to new revolutionary symmetry principles such as mirror symmetry and dualities. S.-T. Yau has played a central role in many of these developments.

Cumrun Vafa Harvard University (Dirac Medal, 2008; Eisenbud Prize 2014; Physics Frontiers Prize 2013)

Calabi-Yau manifolds are used in the context of string theory to make models of particle physics unified with quantum gravity. These models, which were originally constructed in the mid-1980's, were the first reasonably realistic models of particle physics unified with gravity, and-together with variants that have been constructed since, usually with Calabi-Yau manifolds as one of the ingredients-they remain the most interesting candidate models that we have today. In addition, compactification on Calabi-Yau manifolds has given important examples in the study of strong coupling or nonperturbative phenomena in string theory. These examples have been remarkably rich and much insight has come from their study.

Edward Witten

Institute for Advanced Study, Princeton (Fields Medal 1990; Crafoord Prize 2008; Fundamental Physics Prize 2012; Kyoto Prize 2014)

\section{Appendix: Eugenio Calabi}

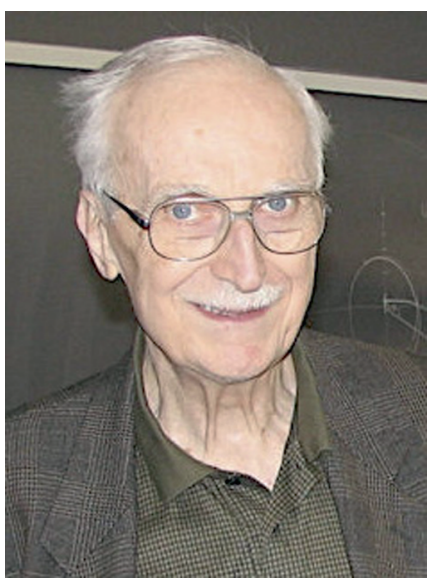

What follows is a brief biography of Calabi with a selective summary of his most important work.

Born 11 May 1923 in Milan, Italy, Calabi earned a Bachelor of Science in chemical engineering from MIT in 1946 and a Master of Arts from the University of Illinois in 1947. He was a Putnam Fellow as an undergraduate at MIT in 1946. He received his Ph.D. degree from Princeton University in 1950 under the supervision of Salomon Bochner. In his dissertation, Calabi developed the theory of isometric complex analytic imbedding of Kähler manifolds.

After graduation, he became an instructor at Princeton University, before joining the faculty of Louisiana State University and later the University of Minnesota. His arrived at the University of Pennsylvania in 1964, was appointed to the Thomas A. Scott Professorship of Mathematics in 1967, and held the post until his retirement in 1993. Calabi's honors include the Guggenheim Fellowship (1962), election to the National Academy of Sciences (1982), the Leroy Steele Prize for Lifetime Achievement in Mathematics (1991) from the AMS, and an Honorary D.Sc. from the University of Pennsylvania (2014).

Calabi made many fundamental contributions to complex differential geometry. In 1950s, he [1] set forth his famous conjecture regarding the existence of Kähler metrics with prescribed Ricci form on Kähler manifolds. The Calabi conjecture was proved by S.-T. Yau two decades later, leading to the creation of Ricci-flat compact Kähler manifolds, now known as "Calabi-Yau manifolds". Calabi-Yau manifolds are higher dimensional analogues of elliptic curves and K3-surfaces, and provide some of the most important and promising Kähler manifolds. They also provide the foundation for string theory, and many believe the universe to have extra dimensions that are curled up in 6-dimensional Calabi-Yau manifolds.

Calabi pioneered the study of rigidity of locally symmetric space. In a very influential paper [8], Calabi and Vesentini proved the rigidity of the complex structure of a compact quotient of a bounded symmetric domain. Calabi [4] also proved deformation rigidity of compact hyperbolic $n$-spaceforms for $n \geq 3$.

Calabi $[2,6]$ also made several pioneering contributions to modern theory of affine differential geometry. Calabi [5] was the first to use ideas of twistor 
theory in the study of minimal surfaces. In [7], Calabi introduced the important concept of extremal Kähler metrics, which is a natural generalization of KählerEinstein metrics. Calabi [3] also constructed some of the most interesting non-Kähler complex manifolds.

\section{References}

[1] E. Calabi, The space of Kähler metrics, Proc. Internat. Congress Math. Amsterdam 2, (1954), 206-207.

[2] E. Calabi, Improper affine hyperspheres of convex type and a generalization of a theorem by K. Jörgens, Michigan Math. J. 5 (1958), 105-126.

[3] E. Calabi, Construction and properties of some 6-dimensional almost complex manifolds, Trans. Amer. Math. Soc. 87 (1958), 407-438.
[4] E. Calabi, On compact, Riemannian manifolds with constant curvature. I, 1961 Proc. Sympos. Pure Math., Vol. III, pp. 155-180. American Mathematical Society, Providence, R.I.

[5] E. Calabi, Minimal immersions of surfaces in Euclidean spheres, J. Differential Geom. 1 (1967), 111-125.

[6] E. Calabi, Complete affine hyperspheres. I, Symposia Mathematica, Vol. X (Convegno di Geometria Differenziale, INDAM, Rome, 1971), pp. 19-38. Academic Press, London, 1972.

[7] E. Calabi, Extremal Kähler metrics, Seminar on Differential Geometry, pp. 259-290, Ann. of Math. Stud., 102, Princeton Univ. Press, Princeton, N.J., 1982.

[8] E. Calabi and E. Vesentini, On compact, locally symmetric Kähler manifolds, Ann. of Math. (2) 71 (1960), 472-507. 